\title{
Baixa dosagem de óxido de cromo para estimativa da produção fecal em bovinos
}

\author{
Low level of chromium oxide to estimate faecal output in cattle
}

\author{
Henrique Mendonça Nunes Ribeiro Filho ${ }^{\text {I* }}$ Francielli Cordeiro Zimermann ${ }^{\text {II }}$ \\ Gilberto Vilmar Kozloski ${ }^{\text {II }}$
}

RESUMO

Foi avaliado o uso de baixa dosagem de óxido de cromo $\left(\mathrm{Cr}_{2} \mathrm{O}_{3}\right)$ incorporado em um alimento concentrado para estimativa da produção fecal (PF) em bovinos. Para tanto, foram conduzidos quatro ensaios de digestibilidade in vivo utilizando quatro novilhos com peso vivo médio de $214 \pm 31 \mathrm{~kg}$, recebendo ad libitum no cocho azevém anual (Lolium multiflorum Lam.) cortado verde. Aproximadamente $200 \mathrm{~g}$ de ração contendo $5 \mathrm{~g} \mathrm{~kg}^{-1}$ do indicador foi fornecida diariamente durante 12 dias, sendo feito a coleta de dados e amostras nos últimos cinco dias de cada período. A PF foi medida com o uso de sacolas de coleção total ou estimada com base na concentração do indicador em amostras fecais coletadas duas vezes ao dia (8 e 16h) ou a intervalos de duas horas entre 8 e 22h. A concentração média de cromo nas amostras coletadas às 8 e 16h (445 $\left.\mathrm{mg} \mathrm{kg}^{-1}\right)$ foi semelhante à média geral de todos os horários (447mg $\mathrm{kg}^{-1}$ ). O grau de recuperação fecal (GR) do indicador aumentou linearmente, de aproximadamente $40 \%$ para em torno de $80 \%$, com o aumento da concentração fecal de cromo até esta última atingir um valor em torno de $250 \mathrm{mg}$ $\mathrm{kg}^{-1}$ de MO. Quando a concentração de cromo nas fezes foi superior a este valor o GR se manteve relativamente constante, em média $76 \%$. A produção fecal foi superestimada pelo indicador em até 35\% quando não corrigido para o GR. Quando corrigido para a recuperação fecal, as estimativas de produção fecal de MO estimadas foram similares às observadas. A excreção fecal de bovinos alimentados com azevém pode ser acuradamente estimada com o uso de baixa dosagem de óxido de cromo incorporado em uma ração peletizada concomitante à coleta de duas amostragens diárias de fezes.

Palavras-chave: bovinos, consumo forragem, Lolium multiflorum Lam.

\begin{abstract}
The aim of this research was to evaluate the use of a low level of chromium oxide $\left(\mathrm{Cr}_{2} \mathrm{O}_{3}\right)$ incorporated into the concentrate ration to estimate faecal output (FO) in cattle. Four in vivo digestibility essays were conducted using four steers with live weights of $214 \pm 31 \mathrm{~kg}$, receiving fresh Italian ryegrass (Lolium multiflorum Lam.). About $200 \mathrm{~g}$ of concentrate with $5 \mathrm{~g} \mathrm{~kg}^{-1}$ of $\mathrm{Cr}_{2} \mathrm{O}_{3}$ was daily rationed during 12 days, where 7 days were used for animal adaptation and 5 days for measurements. The total faecal output was collected and compared with faecal output estimated from grab samples, collected twice a day (8:00 am and 4:00 pm) or at 2-hour intervals, from 8:00 am to 10:00 pm. The average faecal chrome concentration collected at 08:00 am and 04:00 pm (445 $\mathrm{mg} \mathrm{kg}^{-1}$ ) was similar with the average of samples collected at a 2-hour interval (447 $\mathrm{mg} \mathrm{kg}^{-1}$ ). The marker recovery rate $(R R)$ increased linearly, from approximately $40 \%$ to around $80 \%$, with the faecal chrome concentration until approximately $250 \mathrm{mg} \mathrm{kg-1} \mathrm{OM}$. When the faecal chrome concentration was higher than $250 \mathrm{mg} \mathrm{kg}^{-1} \mathrm{OM}$ the RR was relatively constant, with values around 0.76. The faecal output was overestimated up to $35 \%$ when the RR was not considered. When the RR was used, faecal output estimative was similar to the total faecal output collected. The faecal output can be accurately estimated in cattle eating ryegrass using a low level of $\mathrm{Cr}_{2} \mathrm{O}_{3}$ incorporated into the concentrate ration concomitant with twice-a-day faecal grab samples collection.
\end{abstract}

Key words: cattle, herbage intake, Lolium multiflorum Lam.

'Departamento de Produção Animal e Alimentos, Centro de Ciências Agroveterinárias (CAV), Universidade do Estado de Santa Catarina (UDESC). Av. Luiz de Camões, 2090, 88520-000, Lages, SC, Brasil. E-mail: a2hrf@cav.udesc.br. *Autor para correspondência.

"Curso de Medicina Veterinária, CAV/UDESC, Lages, SC, Brasil.

IIIPrograma de Pós-graduação em Zootecnia, Universidade Federal de Santa Maria (UFSM), Santa Maria, RS, Brasil. 


\section{INTRODUÇÃO}

O óxido de cromo $\left(\mathrm{Cr}_{2} \mathrm{O}_{3}\right)$ tem se mantido como um dos indicadores mais utilizados para estimar o consumo de ruminantes em pastejo, o que é justificado, principalmente, pelo seu baixo custo e relativa simplicidade dos procedimentos analíticos (CARVALHO et al., 2007). Contudo, algumas limitações, como recuperação fecal incompleta (SOARES et al., 2004) e irregularidade na excreção ao longo dia (KOZLOSKI et al., 2006; MORENZ et al., 2006), ainda persistem como problemas.

A irregularidade na excreção do $\mathrm{Cr}_{2} \mathrm{O}_{3}$ e a baixa recuperação fecal são atribuídas à sua elevada densidade em relação ao conteúdo do trato gastrintestinal (TGI) (PEYRAUD, 1998). Isso pode ocasionar a formação de sedimento no retículo-rúmen, fazendo com que o indicador seja transferido esporadicamente e dissociado das partículas do TGI (MERCHEN, 1988).

Na tentativa de diminuir a densidade do indicador algumas alternativas têm sido propostas ao longo dos anos. Entre elas destaca-se o uso do $\mathrm{Cr}_{2} \mathrm{O}_{3}$ impregnado a cápsulas de papel, na forma de fibra cromo-mordente ou incorporado em alimento concentrado (PENNING, 2004). O uso do $\mathrm{Cr}_{2} \mathrm{O}_{3}$ misturado em alimento concentrado tem a vantagem de diminuir o estresse causado aos animais durante o fornecimento do indicador. Entretanto, em função de sua baixa palatabilidade, a inclusão de $5 \%$ de $\mathrm{Cr}_{2} \mathrm{O}$ no concentrado, como recomendado por PENNING (2004), tem limitado sua utilização a animais dotados de cânula ruminal (RIBEIRO FILHO et al., 2003). A inclusão do indicador em menores concentrações poderia resolver o problema da aceitação do concentrado pelos animais, desde que não comprometesse a análise do cromo nas fezes.

O objetivo deste trabalho foi avaliar o fornecimento de $\mathrm{Cr}_{2} \mathrm{O}_{3}$ incorporado em um concentrado na proporção de $0,5 \%$ da matéria natural como indicador de produção fecal em animais não canulados.

\section{MATERIAL E MÉTODOS}

Foram realizados quatro ensaios de digestibilidade in vivo utilizando quatro novilhos machos castrados da raça Holandesa com peso vivo médio de $214 \pm 31 \mathrm{~kg}$, mantidos confinados em baias individuais em um galpão coberto. Cada ensaio teve 12 dias de duração, sendo sete de adaptação e cinco de coleta. A forragem utilizada foi o azevém anual (Lolium multiflorum Lam.), com uma idade média de rebrote de 32 dias em todos os ensaios. A forragem foi cortada e fornecida verde, duas vezes ao dia (8h e 16h), ajustando-se diariamente a quantidade para $20 \%$ acima do consumo voluntário do dia anterior.

$\mathrm{O} \mathrm{Cr}_{2} \mathrm{O}_{3}$ foi fornecido incorporado em uma ração peletizada com a seguinte formulação (expressa em $\mathrm{g} \mathrm{kg}^{-1}$ base matéria natural): 400 de casca de soja, 250 de farelo de soja, 200 de farelo de trigo, 100 de melaço em pó, 45 de milho e cinco de $\mathrm{Cr}_{2} \mathrm{O}_{3} \mathrm{~A}$ quantidade oferecida foi de $200 \mathrm{~g}_{\text {animal }}{ }^{-1} \mathrm{dia}^{-1}$, em duas porções de $100 \mathrm{~g}$, fornecidas separadamente da forragem.

As sobras de forragem e eventuais sobras do concentrado eram recolhidas e pesadas diariamente. Amostras representativas foram coletadas, pesadas, secas em estufa com ventilação forçada a $60^{\circ} \mathrm{C}$ por $48 \mathrm{~h}$ e moídas (peneira de $1 \mathrm{~mm}$ ) para posterior análise.

A produção fecal foi medida nos últimos cinco dias de cada período experimental com uso de sacolas de coleção de fezes. Nestes mesmos dias, amostras de fezes foram coletadas diretamente do reto às $8 \mathrm{~h}$ e às $16 \mathrm{~h}$. Para análise, estas amostras foram compostas por animal e tratamento. Para avaliar a flutuação diária da concentração fecal de cromo, amostras de fezes foram coletadas diretamente do reto, em intervalos de duas horas (das 8 às 22h) nos dois últimos dias do primeiro ensaio.

O teor de matéria seca (MS) das amostras foi obtido por secagem em estufa a $105^{\circ} \mathrm{C}$ durante pelo menos 8 horas e, a matéria mineral (MM) pela queima em mufla a $600^{\circ} \mathrm{C}$ durante três horas. O teor de $\mathrm{N}$ total foi determinado por um método Kjeldahl (método 984.13, AOAC, 1995). O teor de fibra em detergente neutro (FDN) foi determinado, sem uso de sulfito de sódio e alfa-amilase, conforme MERTENS (2002). O teor de fibra em detergente ácido (FDA) foi determinado de acordo com o AOAC (método 973.18, AOAC, 1995), mas sem uso de amianto. O cromo nas fezes foi determinado por espectrofotometria de absorção atômica utilizando um método adaptado de WILLIANS et al. (1962), onde a digestão das cinzas resultante de $1 \mathrm{~g}$ de amostra parcialmente seca foi feita com $6 \mathrm{~mL}$ de uma solução ácida $(250 \mathrm{~mL}$ de ácido sulfúrico, $250 \mathrm{~mL}$ de ácido ortofosfórico e $50 \mathrm{~mL}$ de solução sulfato de manganês $10 \% \mathrm{~L}^{-1}$ solução) e $3 \mathrm{~mL}$ de uma solução de bromato de potássio a $4,5 \%(\mathrm{p} / \mathrm{v})$.

A recuperação fecal do cromo foi medida pela relação entre a quantidade de indicador excretado e a quantidade ingerida. A produção fecal (PF) foi estimada pelo indicador como segue: PF (kg de MS $\left.\mathrm{dia}^{-1}\right)=$ indicador ingerido $\left(\mathrm{mg} \mathrm{dia}{ }^{-1}\right) /$ indicador nas fezes ( $\mathrm{mg} \mathrm{kg}^{-1}$ de MS).

Os dados de produção fecal obtidos com o uso das sacolas foram comparados aos estimados pelo 
$\mathrm{Cr}_{2} \mathrm{O}_{3}$ através da análise de regressão. Foram usadas duas regressões lineares para testar a validade do indicador. A primeira $(\mathrm{y}=\mathrm{a}+\mathrm{bX})$ calculou uma intercepta e gerou um $\mathrm{r}^{2}$ que indicou a precisão das estimativas. $\mathrm{O}$ desvio do coeficiente de regressão (b) de 1 foi analisado pelo teste $\mathrm{t}$ bicaudal ( $\mathrm{t}$ calculado $=1$ - b/erro padrão de b). A segunda regressão foi forçada à origem para determinar o desvio (bias) das estimativas ( $\mathrm{y}=\mathrm{bX}$ ). Esta análise foi efetuada com o auxílio do programa computacional GraphPad Prism versão 4.0 Windows ${ }^{\circledR}$, San Diego, USA. Para testar se houve flutuação diária da excreção do cromo, os dados de concentração fecal do indicador nos diferentes horários do dia foram submetidos à análise de variância, que incluiu no modelo o efeito dos animais, ensaios e horário de coleta.

\section{RESULTADOS E DISCUSSÃO}

A concentração fecal de cromo não apresentou diferença significativa $(\mathrm{P}>0,05)$ entre horários de coleta (Figura 1). A média geral de todos os horários foi $447 \mathrm{mg} \mathrm{kg}^{-1}$ de $\mathrm{MO}$, similar à média das amostras coletadas somente às $8 \mathrm{~h}$ e às $16 \mathrm{~h}\left(445 \mathrm{mg} \mathrm{kg}^{-1}\right.$ de MO). Embora a variação entre horários não tenha diferido em amplitudes estatisticamente significativas, a dinâmica de excreção do cromo ao longo do dia foi semelhante à observada por outros autores (MYERS et al., 2006). A variação na excreção fecal do $\mathrm{Cr}_{2} \mathrm{O}_{3}$ ao longo do dia é uma limitação relativamente conhecida (OWENS \& HANSON, 1992) desse indicador e, para contornar esse problema, o uso de várias amostras ao longo do dia tem se constituído em prática que aumenta a confiabilidade dos resultados (OLIVEIRA JUNIOR et al., 2004; KOZLOSKI et al., 2006). Entretanto, no presente estudo o uso de duas amostragens diárias de fezes nos mesmos horários do fornecimento do indicador (8h e 16h) resultou em estimativas de produção fecal similar ao estimado a partir de várias amostragens diárias. Este resultado se deve provavelmente à satisfatória associação do indicador com a digesta pelo fato de ter sido fornecido incorporado a um alimento concentrado suficientemente palatável. Esta condição já foi previamente observada por PEYRAUD (1998) que forneceu óxido de itérbio $\left(\mathrm{Yb}_{2} \mathrm{O}_{3}\right)$ associado a um concentrado peletizado. Além disso, a freqüência diária de fornecimento do indicador também afeta a variação da excreção fecal do $\mathrm{Cr}_{2} \mathrm{O}_{3}$. DETMANN et al. (2001) verificaram que o fornecimento do $\mathrm{Cr}_{2} \mathrm{O}_{3}$ uma vez ao dia subestimou a excreção fecal, enquanto o uso de duas dosagens diárias se mostrou um procedimento mais seguro e acurado.

A recuperação fecal do $\mathrm{Cr}_{2} \mathrm{O}_{3}$, calculada com base em duas amostragens fecais diárias (às 8e 16h), variou em função do teor de cromo nas fezes (Figura 2). Quando a concentração fecal do indicador aumentou de 124 até $250 \mathrm{mg} \mathrm{kg}^{-1}$ de $\mathrm{MO}$, a recuperação fecal aumentou linearmente de pouco mais de 40 para em torno de $80 \%$. Entretanto, não foi influenciada a concentrações fecais do cromo acima de $250 \mathrm{mg} \mathrm{kg}^{-1}$.

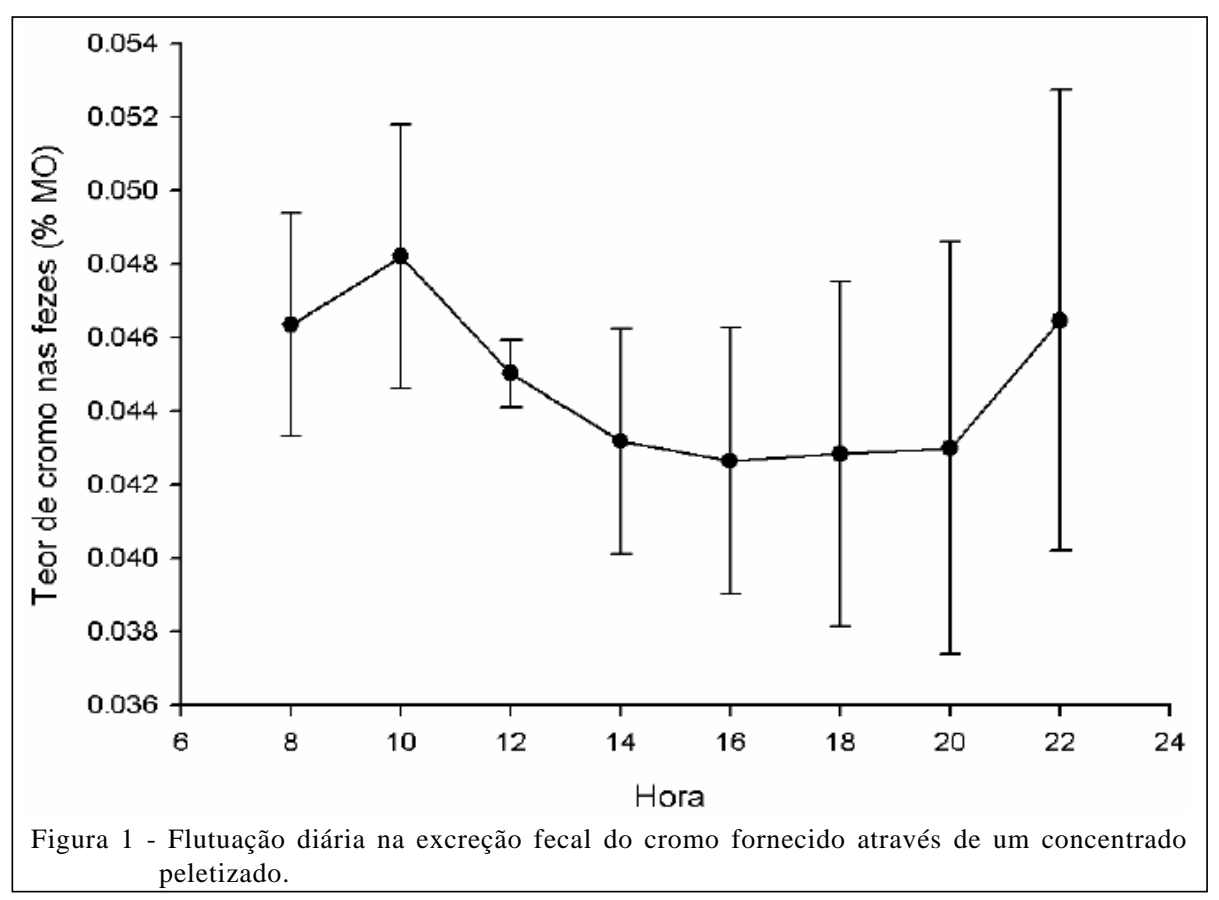

Ciência Rural, v.38, n.9, dez, 2008. 


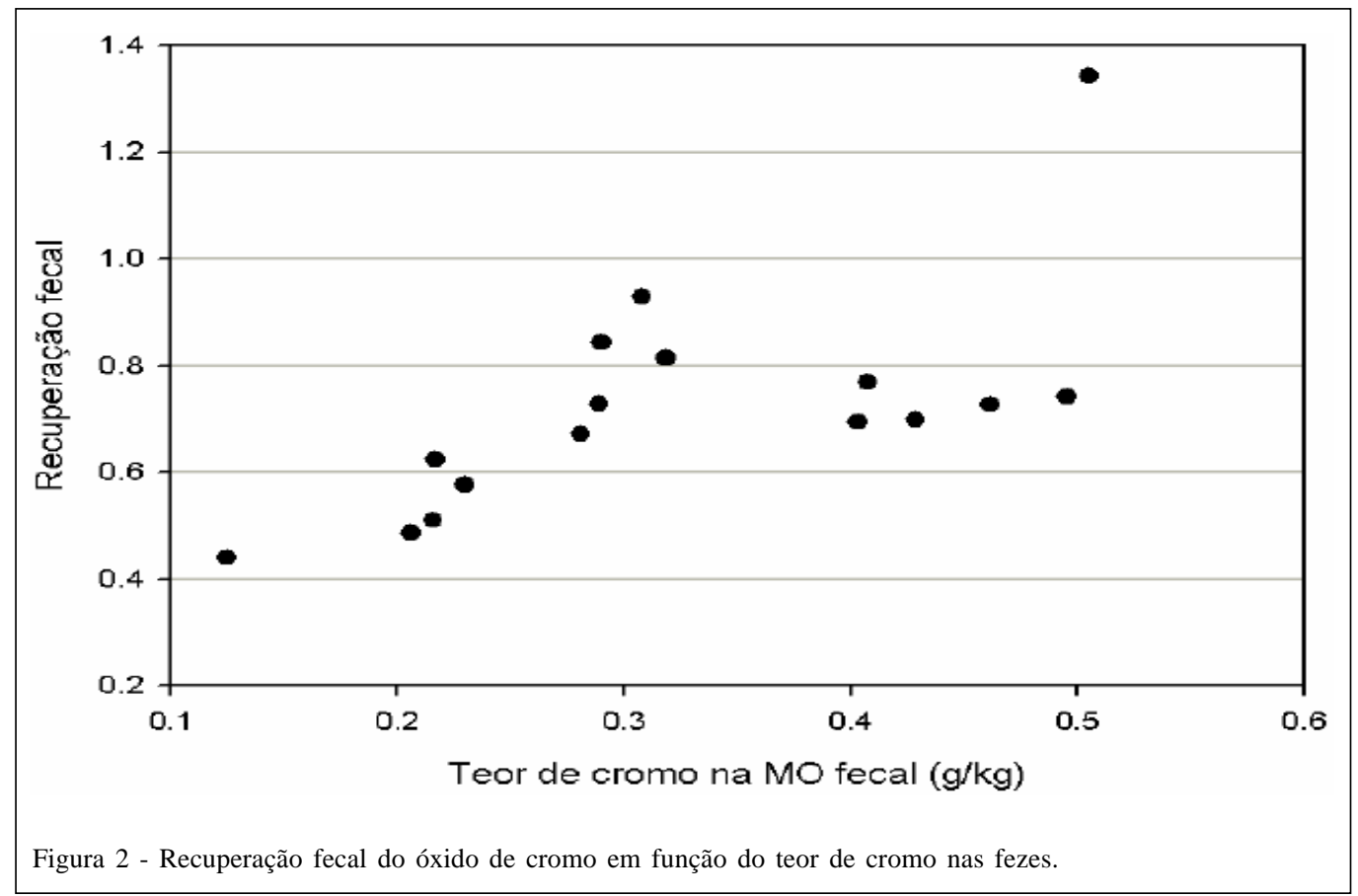

Neste platô a recuperação fecal foi em média 76\%. As concentrações fecais de cromo acima de $250 \mathrm{mg} \mathrm{kg}^{-1}$ de MO foram obtidas nas fezes dos animais que consumiram todo o concentrado, enquanto que as concentrações menores foram correspondentes aos animais que consumiram o concentrado parcialmente. No primeiro caso as quantidades ingeridas de $\mathrm{Cr}_{2} \mathrm{O}_{3}$ foram de $1 \mathrm{~g}$ animal ${ }^{-1} \mathrm{dia}^{-1}$, quantidade cerca de cinco a dez vezes inferior que o normalmente utilizado em outros estudos onde o $\mathrm{Cr}_{2} \mathrm{O}_{3}$ é fornecido em pó por dosagem oral ou via cânula ruminal. A quantidade de cromo fornecida diariamente e, conseqüentemente, sua concentração nas fezes, é frequentemente citada como um dos fatores que pode limitar a validade desta técnica para estimar excreção fecal (OWENS \& HANSON, 1992, PENNING, 2004). Isto ocorre em função do grau limitado de sensibilidade dos métodos laboratoriais usualmente utilizados na análise de cromo. Neste trabalho, nos casos em que a quantidade de indicador ingerida foi abaixo de $1 \mathrm{~g}$ animal ${ }^{-1} \mathrm{dia}^{-1}$, provavelmente o método analítico utilizado não foi sensível o suficiente para detectar adequadamente todo o cromo da amostra.

O valor de $76 \%$ de recuperação fecal do indicador pode ser considerado baixo, uma vez que alguns autores já obtiveram valores médios de recuperação fecal do cromo que vão de 95 a 100\% (LE DU \& PENNING, 1982; MALOSSINI et al., 1996). Entretanto, existem registros na literatura de recuperações fecais de cromo que variam de 75 a $87 \%$ (ZEOULA et al., 2000). Além disso, quando animais ingerem forragens com teores de MS abaixo de 20\%, como o azevém em estágio vegetativo, a coleta total das fezes com uso de sacolas é dificultada em função do alto teor de umidade das mesmas, podendo haver perda de material fecal em torno de até 10\% (MÉLIX \& PEYRAUD, 1987).

Os diferentes valores de taxa de recuperação observados na literatura podem ser atribuídos a distintos protocolos utilizados e a diferenças no tipo e qualidade da dieta. SOARES et al. (2004), por exemplo, observaram maior taxa de recuperação do $\mathrm{Cr}_{2} \mathrm{O}_{3}$ em animais recebendo capim-elefante com 60 dias de rebrote contendo $70 \%$ de FDN do que naqueles recebendo a mesma espécie forrageira com 30 dias de rebrote e 63\% de FDN. De modo similar, LIMA et al (1980) constataram menor uniformidade de excreção, mas maior recuperação fecal do $\mathrm{Cr}_{2} \mathrm{O}_{3}$ em bovinos em pastejo durante o período de estiagem do que no período de chuvas atribuindo este resultado a diferenças no teor de fibra da pastagem (mais alto durante a estiagem) e da sua relação com o fluxo do indicador pelo trato digestivo. OLIVEIRA et al. (1991) e ÍTAVO et al. (2002) também observaram que a redução do teor de fibra da dieta, pela elevação do teor de concentrado, resultou em menor recuperação fecal do óxido de cromo como indicador de excreção fecal. Isto indica que os resultados de flutuação diária da excreção e o grau de recuperação do indicador observados no presente estudo, em que os animais foram alimentados com azevém verde, pode não se aplicar adequadamente a outras condições dietéticas

Ciência Rural, v.38, n.9, dez, 2008. 
Independente do grau de recuperação, a produção fecal dos animais foi estimada com alta precisão $\left(\mathrm{r}^{2}\right.$ em torno de 0,92$)$ pelo uso do cromo adicionado ao concentrado (Figura 3). No entanto, a produção fecal foi superestimada quando a concentração do indicador não foi corrigida para a recuperação fecal (coeficiente de regressão foi maior que $1, P<0,05$ ), mas quando corrigido, os valores estimados foram similares aos observados (coeficiente de regressão não diferiu de 1). Em média, o desvio das estimativas foi de $9,0 \%$ em ambos os casos. A superestimativa na produção fecal, oriunda da
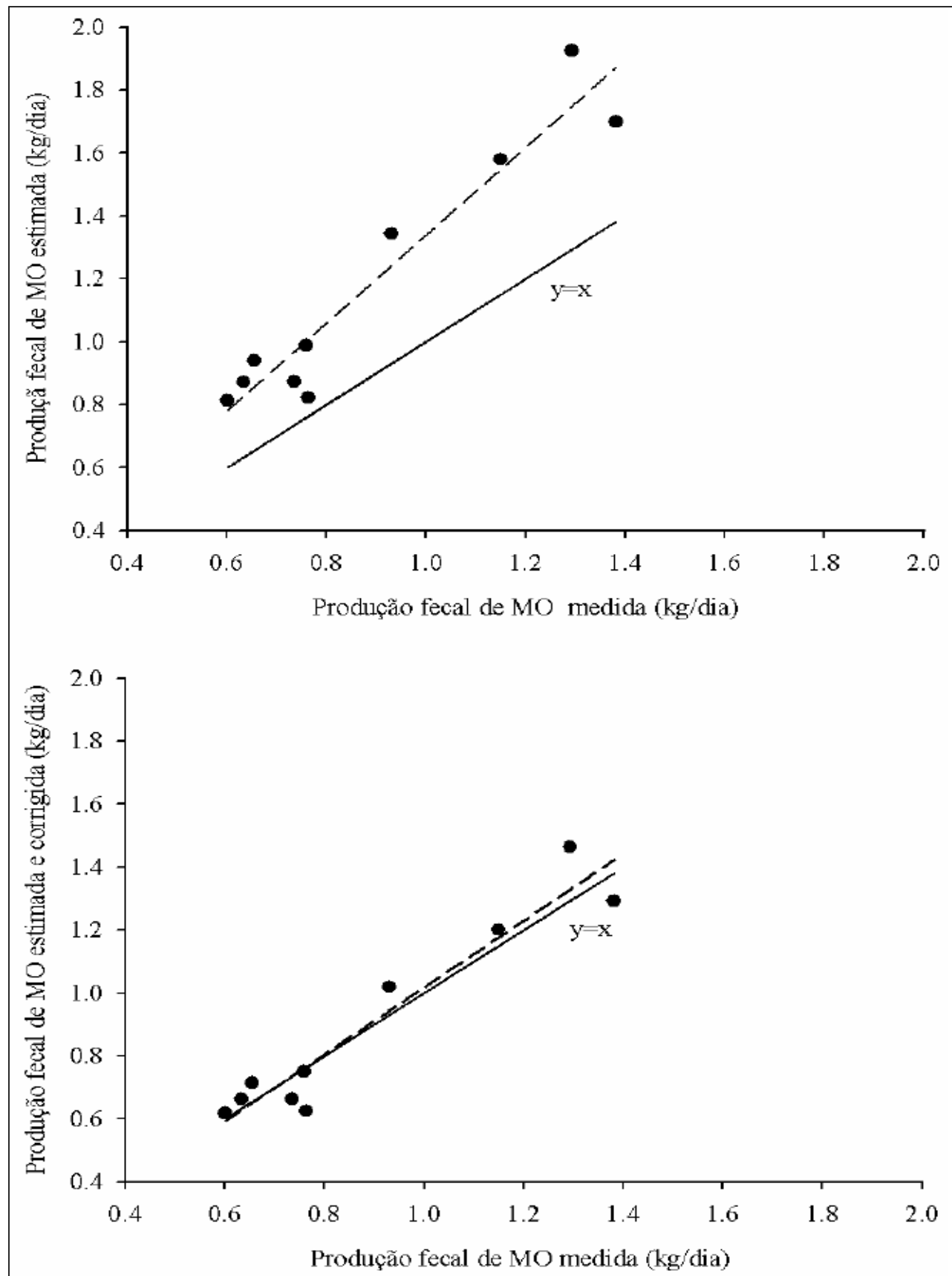

Figura 3 - Relação entre a produção fecal medida e a estimada por diluição de $\mathrm{Cr}_{2} \mathrm{O}_{3}$ considerando recuperação fecal total do indicador $(\mathrm{Y}=1,397 \mathrm{X}-0,0587$, erro padrão da estimativa $=0,126$, $r^{2}=0,92$, o coeficiente de regressão difere significativamente de $1: P<0,05, n=10$ ) ou corrigida para recuperação fecal igual a $0,76(\mathrm{Y}=1,061 \mathrm{x}-0,0446$, erro padrão da estimativa $=0,095$, $\mathrm{r}^{2}=0,92$, o coeficiente de regressão não difere significativamente de $1: \mathrm{P}<0,05, \mathrm{n}=10$ ) .

Ciência Rural, v.38, n.9, dez, 2008. 
recuperação fecal incompleta do indicador é via de regra a principal causa de superestimativa no consumo de forragem em experimentos a pasto (SOARES et al., 1999; 2004). Neste sentido, os resultados do presente trabalho corroboram as recomendações de PENNING et al. (2004) e CARVALHO et al. (2007) indicando que, independentemente do protocolo ou da dieta experimental utilizada, é necessário medir o grau de recuperação fecal do indicador e utilizar este valor no cálculo do consumo diário.

\section{CONCLUSÕES}

O fornecimento do óxido de cromo misturado a um concentrado peletizado, na proporção de $0,5 \%$, concomitante à duas amostragens diárias de fezes, permite estimativas confiáveis da produção fecal de bovinos. Contudo, é recomendado que, para as diferentes dietas e/ou protocolos utilizados, seja medido a taxa recuperação do indicador para correção do cálculo da excreção fecal total.

\section{AGRADECIMENTOS}

Francielli Cordeiro Zimermann agradece a bolsa de iniciação científica, PROBIC/ recebida da UDESC.

\section{REFERÊNCIAS}

ASSOCIATION OF OFFICIAL ANALYTICAL CHEMISTSAOAC. Official methods of analysis. 16.ed. Washington, 1995. 1094p.

CARVALHO, P.C.F. et al. Avanços metodológicos na determinação do consumo de ruminantes em pastejo. Revista Brasileira de Zootecnia, v.36, p.151-170, 2007.

DETMANN, E. et al. Cromo e indicadores internos na determinação do consumo de novilhos mestiços, suplementados, a pasto. Revista Brasileira de Zootecnia, v.30, p.16001609, 2001.

ÍTAVO, L.C.V. et al. Comparação de indicadores e metodologia de coleta para estimativas da produção fecal e fluxo de digesta em bovinos. Revista Brasileira de Zootecnia, v.31, p.18331839, 2002.

KOZLOSKI, G.V. et al. Uso do óxido de cromo como indicador da excreção fecal de bovinos em pastejo: variação das estimativas em função do horário de amostragem. Ciência Rural, v.36, p.599-603, 2006.

LE DU, Y.L.P.; PENNING, P.D. Animal based techniques for estimating herbage intake. In: LEAVER, J.D. (Ed.). Herbage intake handbook. Reading: British Grassland Society, 1982. p.37-75.
LIMA, M.A. et al. O uso do óxido crômico para estimar a produção fecal de novilhos zebu em pastejo. Revista Brasileira de Zootecnia, v.9, p.188-202, 1980.

MALOSSINI, F. et al. Comparison of $n$-alkanes and chromium oxide methods for estimating herbage intake by grazing dairy cows. Animal Feed Science and Technology, v.61, p.155165, 1996.

MÉLIX, C.; PEYRAUD, J.L. Utilisation de l'oxyde de chrome chez les vaches laitières pour la prévision des quantités de fèces émises. I. Etude des variations du taux de récupération et ses conséquences sur l'estimation de la digestibilité et des quantités ingérées de rations d'herbe et d'ensilage de maïs. Reproduction Nutrition Development, v.27, p.215-216, 1987.

MERCHEN, N.R. Digestion, absorption, and excretion in ruminants. In: CHURCH, D.C. (Ed.). The ruminant animal. Digestive physiology and nutrition. Englewood Cliffs, NJ: Prentice-Hall, 1988. p.183-190.

MERTENS, D.R. Gravimetric determination of amylase-treated neutral detergent fibre in feeds with refluxing beakers or crucibles: a collaborative study. Journal of AOAC, v.85, p.12171240, 2002.

MORENZ, M.J.F. et al. Óxido de cromo e n-alcanos na estimativa do consumo de forragem de vacas em lactação em condições de pastejo. Revista Brasileira de Zootecnia, v.35, p.1535-1542, 2006.

OLIVEIRA et al. Estudo da recuperação do $\mathrm{Cr} \mathrm{O}$ e dos indicadores internos CIA, CIDA e lignina em períodos de $^{3}$ coleta de dois a sete dias, em bovinos. Revista Brasileira de Zootecnia, v.20, p.523-531, 1991.

OLIVEIRA JUNIOR, R.C. et al. Avaliação de indicadores para estimar a digestibilidade dos nutrientes em novilhos nelore alimentados com dietas contendo alto teor de concentrado e fontes nitrogenadas. Revista Brasileira de Zootecnia, v.33, p.749-758, 2004.

OWENS, F.N.; HANSON, C.F. External and internal markers for appraising site and extent of digestion in ruminants. Journal of Dairy Science, v.75, p. 2605-2607, 1992.

PENNING, P.D. Animal based techniques for estimating herbage intake. In: PENNING, P.D. (Ed.). Herbage intake handbook. 2.ed. Reading: British Grassland Society, 2004. p.53-93.

PEYRAUD, J.L. Techniques for measuring faecal flow, digestibility and intake of herbage in grazing ruminants. EUROPEAN INTAKE WORKSHOP, 9., 1998, North Wyke. Proceedings... North Wyke: Institute of Grassland and Environmental Research, 1998. p.39-43.

RIBEIRO FILHO, H.M.N. et al. Inclusion of white clover in strip-grazed perennial ryegrass swards: herbage intake and milk yield of dairy cows at different ages of sward regrowth. Animal Science, v.77, p.499-510, 2003. 
SOARES, J.P.G. et al. Capim elefante (Pennisetum purpureum Schum) em pastejo, sob duas doses de nitrogênio. Consumo e produção de leite. Revista Brasileira de Zootecnia, v.28, p.889-897, 1999.

SOARES, J.P.G. et al. Estimativas de consumo de capim-elefante (Pennisetum purpureum Schum), fornecido picado para vacas lactantes utilizando a técnica do óxido de cromo. Revista Brasileira de Zootecnia, v.33, p.811-820, 2004.
WILLIAMS, C.H. et al. Determination of chromic oxide in faeces samples by atomic absorption sectrophotometry. Journal of Agricultural Science, v.59, p.381-385, 1962.

ZEOULA, L.M. et al. Uso de indicadores na determinação da digestibilidade parcial e total em bovinos. Acta Scientiarum, v.22, p.777-777, 2000.

MYERS, W.D. et al. Excretion patterns of titanium dioxide and chromic oxide in duodenal digesta and faeces of ewes. Small Ruminant Research, v.63, p.135-141, 2006. 